\title{
Aspects of Poincaré's Program for Dynamical Systems and Mathematical Physics
}

\author{
Ferdinand Verhulst
}

Received: 11 October 2011 / Accepted: 18 February 2012 / Published online: 10 March 2012

(C) The Author(s) 2012. This article is published with open access at Springerlink.com

\begin{abstract}
This article is mainly historical, except for the discussion of integrability and characteristic exponents in Sect. 2. After summarising the achievements of Henri Poincaré, we discuss his theory of critical exponents. The theory is applied to the case of three degreesof-freedom Hamiltonian systems in $(1: 2: n)$-resonance $(n>4)$. In addition we discuss Poincaré's mathematical physics, in particular the theory of partial differential equations, rotating fluid masses and relativity. Attention is given to the priority question of Special Relativity.
\end{abstract}

Keywords Henri Poincaré · Dynamical systems · Characteristic exponents · Rotating fluids · Relativity

\section{Key Results of Henri Poincaré}

For most of us, understanding a new topic in mathematics or physics and subsequently extending it substantially, is quite an achievement. It is truly amazing that Henri Poincaré developed whole new fields from scratch, and, in the case that other people started earlier on the subject, that he gave an essentially new approach to the problems after which the research field was never the same again. The basic results of Henri Poincare in pure and applied mathematics, physics and around science include the following topics:

1. Automorphic functions, uniformisation (involving Riemann surfaces and non-Euclidean geometry).

2. The qualitative theory of differential equations with its emphasis on global understanding of the flow of solution in suitable spaces.

3. Asymptotic expansions, normal forms that provide a quantitative complement to the qualitative theory.

4. Dynamical systems including bifurcation theory and integrability of Hamiltonian systems.

F. Verhulst (ه)

Department of Mathematics, Utrecht University, P.O. Box 80.010, 3508 TA Utrecht, The Netherlands

e-mail: f.verhulst@uu.nl 
5. Mathematical physics that ranges from the abstract theory of partial differential equations to topics like Maxwell's electromagnetic theory and cosmogony.

6. Topology (analysis situs) with its extension of the Euler characteristic to smooth manifolds, the concept of homology and the Poincare conjecture.

7. Philosophy in which conventionalism in mathematics gives the freedom to develop different but in itself consistent mathematical theories; in addition the notion that an axiomatic construction of mathematics based on logic can never be complete as intuition is essential.

In his 1913 'éloge', Darboux [1], considered the topic of automorphic functions Poincaré's finest achievement. It is clear that its combination of (complex) analysis and geometry appealed strongly to Darboux, it fitted with his own style of doing mathematics. Automorphic functions is a beautiful and difficult part of complex function theory with still fundamental open questions regarding the theory of more than one complex variable. But nowadays it is not so easy to give a preference to one of Poincaré's theories as during the last hundred years the focus of mathematics and physics shifted a number of times. However, we can safely say that many of Poincaré's papers and ideas opened new vistas. In this article we will only consider a few aspects of his work, the literature on the achievements of Poincaré's work is enormous; for more details and many more references see [26]. Regarding references for modern results on Hamiltonian systems see [21, Chap. 10].

\subsection{Differential Equations and Dynamical Systems}

The results in this field can be found in various papers and in the 'Méthodes Nouvelles de la Mécanique Célèste' [19]. The term 'Méthodes Nouvelles' indicates a new approach with respect to the classical methods of Lagrange, Laplace, Delaunay and Jacobi. The new methods from the papers and the books include:

- Index theory.

- The Poincaré-Bendixson method for periodic solutions.

- The Poincaré-Lindstedt expansion method as continuation method and as bifurcation method for periodic solutions.

- Characteristic exponents and expansion of exponents in the presence of a small parameter; exponents when first integrals exist.

- The famous proof that in general for time-independent Hamiltonian systems no other first integrals exist besides the energy.

- The divergence of series expansions in celestial mechanics.

- The recurrence theorem.

- Homoclinic (doubly asymptotic) and heteroclinic solutions; the image of the corresponding orbit structure.

In this long list, the first two items obtained a complete form in the papers of Poincaré, the other items have seen a long development after him. In our paper we will only consider in Sect. 2 the theory of critical exponents with its relevance for modern integrability research.

\subsection{Mathematical Physics}

Poincaré is known nowadays among applied mathematicians and physicists mostly for his work on dynamical systems, including celestial mechanics, but his results on mathematical physics are definitely of fundamental importance. We first mention the spectral theory of partial differential equations which precedes the work of David Hilbert. As we shall see, he 
was also the first to introduce the concept of a generalised function and, inspired by work of Tchebytcheff, convergence in (what we now call) $L^{2}$-norm. Rotating fluid masses in relation to the cosmogonic theories of that time got his attention; these papers and books are still of interest, partly because of the mathematical results, but also because of the lucid assessment of contemporary work. At the end of the nineteenth century, the discussion started of the electromagnetic field theory of Maxwell, the theory of the electron and the atom, the new concepts of mass, space and time developed by Hendrik Lorentz. Poincaré contributed extensively to these discussions, for instance with his introduction of the Poincaré group for Lorentz transformations and the formulation of the principle of relativity. We shall describe part of this in Sect. 3; see again [26] for more details.

\section{Critical Exponents}

To study the stability of equilibria of differential equations, we can compute eigenvalues of a matrix obtained by linearisation near such an equilibrium. For time-dependent solutions, Poincaré and independently Lyapunov, came up with a more general concept. These are the characteristic exponents introduced in chapter four of the 'Méthodes Nouvelles'. Consider an $n$-dimensional autonomous equation of the form

$$
\dot{x}=X(x),
$$

and suppose we know a particular solution $x=\phi(t)$. We call this a generating solution, it will usually be a periodic solution. When studying neighbouring solutions of $\phi(t)$ we put

$$
x=\phi(t)+\xi .
$$

The variational equations of $\phi(t)$ are obtained by substituting $x=\phi(t)+\xi$ into the differential equation and linearising for small $\xi$ to obtain

$$
\dot{\xi}=\left.\frac{\partial X}{\partial x}\right|_{x=\phi(t)} \xi .
$$

If $\phi(t)$ is a periodic solution, the variational equations are a Floquet system. In what follows we assume periodicity.

The variational equations are forming a linear system of equations with periodic coefficients of which the characteristic eigenvalue equation produces the characteristic exponents. Poincaré develops conditions for the characteristic exponents that are leading to periodic solutions bifurcating from $\phi(t)$.

In addition, there is a relation between characteristic exponents and integrability in the case of Hamiltonian systems.

- It is clear from the linear system determining the characteristic exponents that in the autonomous case, the periodic function $\dot{\phi}(t)$ is a solution, so one of the characteristic exponents is zero.

- If the vector field $X$ is autonomous and we have $p$ independent first integrals, $p<n$, we have at least $p+1$ characteristic exponents zero.

A number of special results hold in the case that our nonlinear system of differential equations is Hamiltonian and autonomous. Poincaré proves, that in this case the $2 n$ characteristic exponents of a periodic solution, emerge in symmetric pairs $\lambda_{i},-\lambda_{i}$, equal in size and of opposite sign. In addition, the energy integral produces a characteristic exponent zero so we have for a periodic solution of a time-independent Hamiltonian system two characteristic 
exponents zero; if there exist $p$ independent first integrals we have either $2 p+2$ characteristic exponents zero or, in the exceptional case, the functional determinants of the integrals restricted to the periodic solution vanish. For the proof, Poincaré uses Poisson brackets and the theory of independent solutions of linear systems.

\subsection{Integrability Derived from Characteristic Exponents}

One can try to turn the argument around. If we find for a time-independent Hamiltonian system a periodic solution with more than two zero characteristic exponents, this can be caused by the presence of another first integral besides the energy or it may be the exceptional case.

The technical problems connected with drawing conclusions from the presence of more than two zero characteristic exponents, have probably prevented its general use in research of conservative dynamics, but the statement "a continuous family of periodic solutions on the energy manifold is a non-generic phenomenon" is one of the remaining features in the literature. Nowadays the analysis of characteristic exponents is made easier by the use of numerical continuation methods.

\subsection{Integrability of Hamiltonian Normal Forms}

The normal forms of two degrees-of-freedom time-independent Hamiltonian systems near stable equilibrium are always integrable, so these cases are in this respect less interesting. Examples of more than two zero characteristic exponents are often found in the normal forms of three degrees-of-freedom systems, for instance in the $1: 2: n$-resonance with $n>4$, where normalisation to the cubic part of the Hamiltonian written in the usual $(p, q)$ coordinates produces two families of periodic solutions on the energy manifold. Such cases are studied in [24]. The normal form truncated to cubic terms is integrable. The families break up when adding higher order normal form terms. More explicitly, consider the Hamiltonian in action-angle variables $\tau, \phi$ and integer $n$ :

$$
H(\tau, \phi)=\tau_{1}+2 \tau_{2}+n \tau_{3}+H_{3}+H_{4}+\cdots \quad n>4 .
$$

In the three degrees-of-freedom Hamiltonian written in $(p, q)$ coordinates, the cubic part has 56 terms, the quartic part 126 terms. In action-angle coordinates, the degrees change, but we keep referring to $\mathrm{H}_{2}, \mathrm{H}_{3}$ etc. where the index gives the degree for $(p, q)$ coordinates. The normal form is indicated by a bar. Calculating the normal form (see $[21,24])$ of the cubic terms, we find if $n>4$ :

$$
\bar{H}_{3}(\tau, \phi)=4 a_{1} \tau_{1} \sqrt{\tau_{2}} \cos \left(\xi_{1}\right),
$$

where we have the combination angle $\xi_{1}=2 \phi_{1}-\phi_{2}-a_{2}$, the real constants $a_{1}, a_{2}$ are the only constants surviving the normalisation process. The action simplex of the normal form $H_{2}+\bar{H}_{3}$ is displayed on the left in Fig. 1. There are two families of periodic solutions branching off the $\tau_{3}$-normal mode on the energy manifold, each of them producing four zero characteristic exponents and two nonzero ones. This normal form is integrable as the third degree of freedom is decoupled at this level of approximation; the independent integrals are $H_{2}+\bar{H}_{3}, H_{2}$ and $\tau_{3}$. In three degrees-of-freedom Hamiltonian systems, there are at least two characteristic exponents zero; $O$ indicates two extra characteristic exponents zero, $H$ indicates hyperbolic behaviour, $E$ elliptic.

Adding the normal form $\bar{H}_{4}$ of the quartic terms changes the picture for $n=5,6$. If $n>6$ the normal form to $H_{4}$ remains integrable. For illustration we give the normal form for $n=5$ and the corresponding action simplex on the right in Fig. 1. The two families 


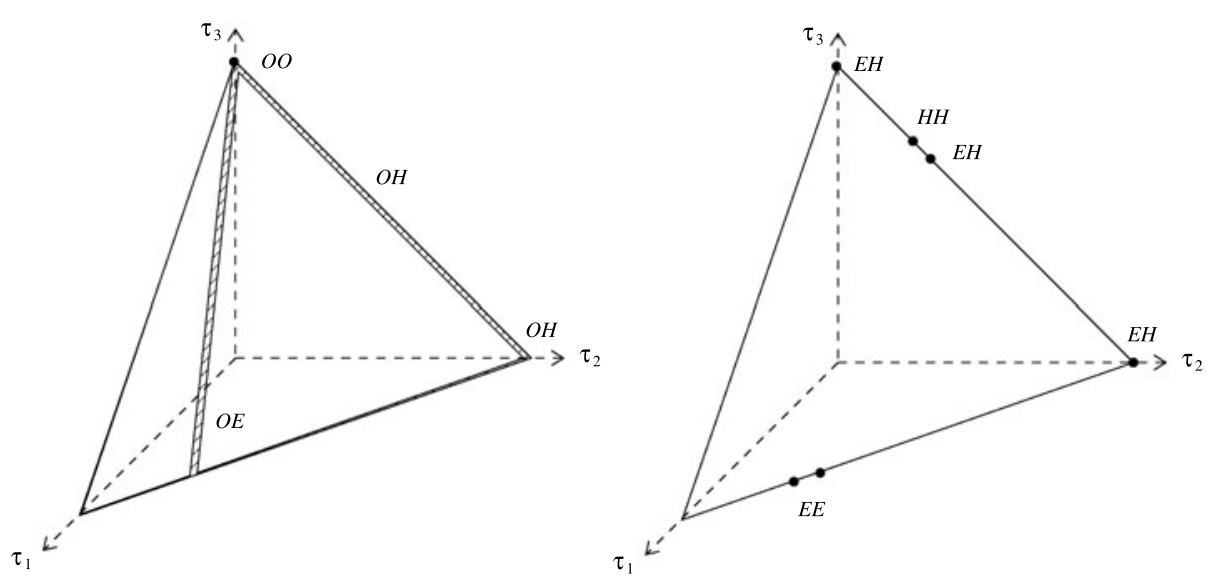

Fig. 1 The three degrees of freedom $(1: 2: n)$-Hamiltonian resonance with $n>4$; left the periodic solutions in an action simplex of the Hamiltonian normalised to $H_{3}$, indicated by a $d o t$ (the $\tau_{3}$-normal mode) and the hatched strips for families of periodic solutions; on the right the results of normalisation to $\mathrm{H}_{4}$ in the case $n=5$, see [21] (figure courtesy Springer)

of periodic solutions break up into isolated periodic solutions. Introducing the combination angle $\xi_{2}=\phi_{1}+2 \phi_{2}-\phi_{3}-b_{8}$ we find with real constants $b_{1}, \ldots, b_{8}$ :

$$
\bar{H}_{4}(\tau, \phi)=4\left(b_{1} \tau_{1}^{2}+b_{2} \tau_{1} \tau_{2}+b_{3} \tau_{1} \tau_{3}+b_{4} \tau_{2}^{2}+b_{5} \tau_{2} \tau_{3}+b_{6} \tau_{3}^{2}+b_{7} \tau_{2} \sqrt{\tau_{1} \tau_{3}} \cos \xi_{2}\right) .
$$

It is possible that chaotic behaviour can be demonstrated in a three degrees-of-freedom Hamiltonian normal form. An indication for this can be the presence of a periodic solution with complex eigenvalues; in such a case this can lead to Shilnikov-Devaney bifurcation. From the simplex in Fig. 1, it is clear that this case does not present itself in the $(1: 2: 5)$ resonance; from [24] it follows that also the $(1: 2: 6)$-resonance has no complex eigenvalues with nonzero real parts. The only remaining candidate for this behaviour is the $\tau_{3}$-normal mode in the $(1: 2: n)$-resonance with $n>6$.

We note finally, that it is remarkable that we have an exceptional case for the general $(1: 2: 2)$-resonance, which is a first order resonance. First, the cubic normal form turns out to be integrable with respectively energy integral, a quadratic integral and a cubic integral. Moreover, on the energy manifold we find one continuous family of periodic solutions and two isolated solutions. So we have here an exceptional case as described by Poincaré. The resonance is studied in [25].

\section{Mathematical Physics}

In this section we will briefly describe methods developed by Poincaré for partial differential equations and in the following subsections a number of physical theories. We aim at conveying the ideas while leaving technical details to the literature cited.

\subsection{Partial Differential Equations}

The survey [10] gives details, for instance noting the first instance where the idea of a generalised solution is formulated, and ends with the conclusion: 
Poincaré's contributions to the equations of mathematical physics would have sufficed to place him among the greatest mathematicians of the end of the 19th and the beginning of the 20 th century.

In 1890, Poincaré [12] observed how similar the equations are from very different fields of physics and chemistry. Considering the static or dynamic theory of electricity, optics, the theory of heat, elasticity or hydrodynamics, one is always led to the study of the same group of differential equations with primary example the Laplace equation. Also the boundary conditions that are supplementing the equations show this similarity. This forms a good reason for paying special mathematical attention to these typical equations.

\subsubsection{The Balayage or Sweeping Method}

When considering the Newtonian attraction properties of bodies with a given distribution of mass, one is led to a study of the Laplace and Poisson equations. One of the basic problems is to solve the equation

$$
\Delta V=\frac{\partial^{2} V}{\partial x^{2}}+\frac{\partial^{2} V}{\partial y^{2}}+\frac{\partial^{2} V}{\partial z^{2}}=0 \quad \text { in } D,
$$

with $D \in \mathbb{R}^{3}$ a bounded domain. A twice differentiable function satisfying the Laplace equation $\Delta V=0$ is called harmonic. If we require that on the boundary $S$ of $D$ we have $V=\Phi$ with $\Phi$ a known function, this is called the Dirichlet boundary value problem for the Laplace equation.

An elegant method to solve the Dirichlet boundary value problem is to consider the Dirichlet functional

$$
I(V)=\int_{D}\|\nabla V\|^{2} d x d y d z
$$

with $V$ an element of the set of twice differentiable functions on $D$ that are continuous on $D \cup S$. The nabla operator $\nabla$ produces the gradient of $V,\|$.$\| is the Euclidean norm.$ The Dirichlet principle states that if one minimises the functional $I(V)$ over the subset of functions $V$ that satisfy the boundary condition, this solves the boundary value problem.

Around 1890, the validity of the Dirichlet principle was not yet proved, indeed Weierstrass had thrown doubt on it, so scientists were looking for alternative solution methods.

Poincaré's balayage or sweeping method was published in [12], a didactical presentation is given in his lecture notes Théorie du potentiel Newtonien (1899). In the lecture notes, the balayage method is explained for the interior of a sphere after which more complicated geometries can be studied. The method uses that the potential of a mass distribution will not change when we replace each element of mass in the sphere by a mass layer on the surface with appropriate density. The mass distribution on the surface is called the equivalent layer. With this procedure we perform a sweeping (balayage) of all the mass in the interior.

To consider, more in general, a bounded, connected domain with smooth boundary surface, Poincaré uses a covering of the domain by a denumerable set of balls and a corresponding sequence of harmonic functions.

In modern potential theory one considers domains in $\mathbb{R}^{n}$ with Borel measures (instead of mass distributions) on sets of a general nature where the balayage produces another suitable measure. Nowadays, the balayage method plays a part only in abstract potential theory. 


\subsubsection{Spectral Analysis}

Consider an evolution equation on a bounded domain $D \in \mathbb{R}^{3}$ with smooth boundary $S$ and a Dirichlet boundary condition $\left.\phi\right|_{S} ; t \geq 0$. Initially, when $t=0$, we prescribe $\phi(x, y, z, 0)$. When looking for solutions in a form that separates space and time, we often find an equation on $D$ with spatial derivatives only:

$$
\Delta U+k U=0 .
$$

The boundary condition, if it is linear, also separates; we choose the mixed condition

$$
\frac{\partial U}{\partial n}+h U=0 \quad \text { on } S .
$$

$\partial U / \partial n$ is the exterior normal derivative, $h$ is a given constant. For the real number $k$ we will find an infinite, denumerable set of values such that $U$ satisfies the boundary condition. We indicate these solutions by $U_{k}$, the orthogonal eigenfunctions, the corresponding numbers $k_{1}, k_{2}, \ldots$ are called the eigenvalues. One usually normalises

$$
\int_{D} U_{k}^{2} d x d y d z=1
$$

Any linear combination of eigenfunctions will solve the spatial boundary value problem, the separation process is called separation of variables or Fourier analysis. In Poincaré's time, the spectral analysis of such eigenvalue problems was not rigorous, except for certain simple geometries of the domain $D$.

A first step is estimation of the eigenvalues by variational techniques. In the period 18871890 Poincaré formulated an extension of the Dirichlet principle, see [12]. Consider the expression

$$
B(F)=h \int_{S} F^{2} d \sigma+\int_{D}\|\nabla F\|^{2} d x d y d z
$$

and the normalisation condition

$$
A(F)=\int_{D} F^{2} d x d y d z=1 .
$$

The method of Lagrange multipliers gives us that the first eigenfunction $U_{1}$ with corresponding eigenvalue $k_{1}$ minimises $B(F)$ over the set of nontrivial $C^{1}$-functions that satisfy $A(F)=1$. Moreover we have

$$
k_{1} \leq \frac{B(F)}{A(F)}
$$

for all nontrivial functions $F$. Minimising $B(F)$ over the smaller set of functions satisfying

$$
\int_{D} F^{2} d x d y d z=1, \quad \int_{D} F U_{1} d x d y d z=0,
$$

we find the second eigenfunction $U_{2}$ with eigenvalue $k_{2}$. This minimalisation process can be continued. In addition Poincaré also obtained an upper bound for the eigenvalues. Introducing $F$ as a linear combination

$$
F=\sum_{j=1}^{n} \alpha_{j} F_{j}
$$


and introducing the set $S_{n}$ over which will be minimalised:

$$
S_{n}=\left\{\sum_{j=1}^{n} \alpha_{j} F_{j}: \alpha \in \mathbb{R}^{n}\right\},
$$

it follows that we have the minimax characterisation of eigenvalues:

$$
k_{n}=\min _{S_{n}} \max _{F \in S_{n}} \frac{B(F)}{A(F)} .
$$

The last result is implicit in his calculations. By considering lower bounds for the eigenvalues, Poincaré could show that $k_{j} \rightarrow \infty$ as $j \rightarrow \infty$.

\subsubsection{The Poincaré Inequality}

An important generalisation by Poincare of the estimates on eigenvalues is to give bounds on a function in terms of its derivatives and the geometry of the domain of definition; such bounds are nowadays formulated in norms corresponding with Sobolev-spaces. A typical result for a continuously differentiable function $V$ defined on a convex set $D$ in threedimensional space such that $\int_{D} V d x d y d z=0$ is

$$
\int_{D} V^{2} d x d y d z \leq c \int_{D}\|\nabla V\|^{2} d x d y d z
$$

with suitable, but at this stage unknown constant $c$.

In studies appearing after Poincaré, the estimates were extended for eigenvalue problems in $\mathbb{R}^{n}$, optimal values for the numerical constants have been obtained.

\subsubsection{Existence Questions of Spectral Analysis}

The proof of the existence of an infinite number of eigenvalues and eigenfunctions for the Dirichlet problem of elliptic equations like (1), is usually attributed to Fredholm and Hilbert. A first proof however, was given in 1894 by Poincaré in $[13,14]$. Consider the heat equation $\partial \phi / \partial t=\Delta \phi$ on a bounded domain $D \in \mathbb{R}^{3}$ with smooth boundary $S$ and boundary condition $\partial U / \partial n+h U=0 ; h$ represents the emission coefficient of heat from the surface. Assuming that the body contains a source of heat, the equation is modified to

$$
\frac{\partial \phi}{\partial t}=\Delta \phi+q
$$

where we have to specify $q$ as a function of space and time. With simplifying assumptions the separated equation becomes

$$
\Delta U+\xi U+f=0 \quad \text { in } D, \quad \frac{\partial U}{\partial n}+h U=0 \quad \text { on } S,
$$

with $f$ a constant, $\xi$ a parameter. Following the approach of Schwarz, Poincaré expands the solution in powers of $\xi$ :

$$
U=U_{0}+\xi U_{1}+\xi^{2} U_{2}+\cdots .
$$

For the coefficients one obtains the sequence of equations

$$
\Delta U_{0}+f=0, \quad \Delta U_{n}+U_{n-1}=0, \quad n=1,2, \ldots .
$$

The boundary conditions for the $U_{n}$ are inherited from the boundary condition for $U$. Considering first the Dirichlet problem $U=0$ on $S$, one can construct the $U_{n}$, using the Green's 
function for the Laplace operator. From this integral expression, one can estimate the $U_{n}$ to conclude that the series converges absolutely and uniformly with positive radius of convergence in $\xi$. In the construction, functions $P_{k}$ arise that are called harmonic; they satisfy the equation

$$
\Delta P_{k}+k P_{k}=0,
$$

$k$ is called characteristic number. Nowadays we call $P_{k}$ eigenfunction and $k$ corresponding eigenvalue.

\subsubsection{Convergence in the Mean}

In the lecture notes Théorie analytique de la propagation de la chaleur, (1893-1894), classical convergence proofs of series expansions for non-stationary heat flow are based on methods devised by Cauchy. In [13, Chap. 3], Poincaré notes the following problem. Consider again the boundary value problem for a cooling body in the form

$$
\frac{\partial V}{\partial t}=\Delta V \quad \text { in } D, \quad \frac{\partial V}{\partial n}+h V=0 \quad \text { on } S .
$$

At the initial time, say $t=0$, the temperature $V=V_{0}(x, y, z)$ is given and, using the derivatives at $t=0$, we can formulate an expansion in powers of $t$. It is strange that the shape of the domain $D$ does not enter in this expansion. To be more explicit, in the one-dimensional case with Neumann conditions we have:

$$
V(t, x)=\sum_{m=0}^{\infty} A_{m}(t) \cos m x .
$$

The function will in general be discontinuous near the boundary, the expansion with respect to powers of $t$ obtained above, seems to make no sense. Poincaré was at this point inspired by Tchebytcheff [22], who developed orthogonal polynomial expansions to solve a very different kinematical, geometric problem, but who found no convergence of his expansions. Tchebytcheff 'solved' his problem by requiring the error of his expansion to satisfy a minimal value in the sense of least squares. In a similar way, Poincaré, referring to Tchebytcheff, required the solution of the cooling problem to have a small average error $S(t)$ at time $t$ by considering:

$$
S(t)=\int_{D}\left(V(t, x)-\sum_{m=0}^{N} A_{m}(t) \cos m x\right)^{2} d x,
$$

that becomes smaller as $N$ increases. This convergence in the mean is now called convergence in $L^{2}$-norm.

\subsection{Rotating Fluid Masses}

In the 18th century, scientists became interested in the equilibrium shapes of rotating fluid masses under the action of their own gravity and the fluid pressure that is present; such fluids are called self-gravitating. The interest arose from discussions about the shape of planets and stars. The underlying physical assumptions were far removed from modern insights like internal energy production, internal motions and dissipative effects, so the modelling is too simple for modern astrophysics, but the results and analysis are still basically of mathematical and even some astrophysical interest. A relatively recent survey of the literature can be 
found in [5], for Poincaré's results we will use his lecture notes Figures d'équilibre d'une masse fluide (1902). One of his earlier publications was in the Acta Mathematica for 1885. Poincaré's interest was triggered by an idea put forward by William Thomson (Lord Kelvin) who observed that our planetary system consists of many bodies and also that star systems seem to be more often multiple than not; he then made the hypothesis that such multiplicity arose from fission of rotating fluid masses. A possible mechanism for such an instability would be small friction, see also the classic monograph of Thomson and Tait [23] or the modern survey [3].

When linearising around an equilibrium state to determine its stability, one calculates the eigenvalues. If these are all purely imaginary, the equilibrium was called 'ordinary stable', in modern times this is called 'neutrally stable' or 'Lyapunov-stable'. If all the eigenvalues have real parts negative, it was called 'secularly stable', this is now called 'asymptotically stable'. In the following we will keep to the modern terminology.

\subsubsection{Classical Results}

Without rotation, the sphere gives the stable equilibrium solution. Maclaurin (1698-1746) derived an explicit expression for a rotating fluid mass that is an oblate spheroid. The flattening (eccentricity) depends on $\omega$, the angular velocity around a fixed axis of rotation through the centre; the rotation of the fluid mass is assumed to be solid. Later, the results of Maclaurin were generalised to ellipsoids with all axes unequal, the so-called triaxial Maclaurin ellipsoids.

Jacobi (1804-1851) assumed a slightly simpler expression for the potential and found a second family of triaxial ellipsoids that is independent of Maclaurin's ellipsoids. Interestingly, there is one point in parameter-space where they coincide, a bifurcation point of the families of ellipsoids.

Both families represent special solutions of solid mass rotation with corresponding density distributions, eccentricities and rotational velocities.

Important steps forward were made by successively Dirichlet (1805-1859), Dedekind (1831-1916) and Riemann (1826-1866). They started with the partial differential equations of fluid mechanics to find solutions by a similarity approach. Assuming a certain spatial structure leaves equations to solve as functions of time; Dirichlet could solve the equations in the case of a homogeneous ellipsoid. Dedekind gave more details of Dirichlet's model and added an ellipsoid that is characterised by motions of constant vorticity. Riemann in his turn clarified these models and added stability considerations.

\subsubsection{Poincaré's Contribution}

The fission hypothesis of Thomson was to consider the evolution of steady state solutions, like the rotating Maclaurin ellipsoids with increasing rotational velocity, that at some stage of evolution would split into two equilibrium figures. The mechanism to produce such a bifurcation could be rotation or dissipation-induced instability caused by the small viscosity of the fluids. Among the scientists who studied this scenario assuming solid body rotation, were Lyapunov and Poincaré; the earlier results on ellipsoids with internal dynamics were largely ignored.

In Poincaré's lecture notes Figures d'équilibre d'une masse fluide (1902), the theory is developed from first principles, starting with Newtonian gravitational attraction, defining the gravitational potential $\Phi$ and stating the usual basics of potential theory, the Laplace equation, the Poisson equation, the theorems of Gauss and Green. Following the analysis of 
Maclaurin and Jacobi, a homogeneous fluid is considered. In this case the constant angular velocity $\omega$ has an upper limit, a necessary condition for equilibrium is in suitable physical units:

$$
\omega^{2} \leq 2 \pi
$$

For the two types of triaxial ellipsoids attributed to Maclaurin and Jacobi, Poincare derives the conditions $\omega<4 \pi \times 0.112$ and $\omega<4 \pi \times 0.093$.

According to Dirichlet, for an equilibrium to be stable, a certain energy functional has to be maximal. Thomson [23] observed that the equilibrium is in this case also asymptotically stable if we add friction. If an equilibrium, corresponding with a stationary point of the functional, does not maximise the expression, it can be Lyapunov-stable, but in this case it may be unstable with respect to dissipative effects. Poincaré shows that in the case of triaxial ellipsoids, stable equilibrium requires rotation about the smallest axis.

In Poincaré's lecture notes considerable attention is paid to suitable orthogonal special functions. These are the spherical functions, polynomials derived in polar coordinates from the Laplace equation, and the Lamé functions, polynomials derived in ellipsoidal coordinates. The Lamé functions play a prominent part in the expansions for Maclaurin's and Jacobi's triaxial ellipsoids. At this point, using higher order Lamé functions, Poincaré discovered a new series of solutions branching off the Jacobi ellipsoids. He called them 'pearshaped'.

If the constriction of the pear-shaped figures would narrow during evolution, Poincaré expects cooling of the fluid; such slowly evolving equilibria would be suitable candidates for the fission theory of planetary and stellar systems. His stability analysis depended on linearisation of the equations and was not completed during his life-time.

Regarding the stability of the new equilibrium figures found by Poincaré, the pear-shaped figure (which he thought very promising) turned out to be unstable, but some of his other solutions, emerging from higher order harmonics, show interesting aspects. Paul Appell was one of the contributors to solving the stability problems of the pear-shaped figures. For the developments since Poincaré's time, see [5].

Poincaré introduced an interesting result that plays a part in general bifurcation theory: the phenomenon of 'exchange of stabilities'. For instance in the so-called transcritical bifurcation, two solutions exist if a certain parameter $\mu$ is smaller and larger than a critical value. At the critical value they coincide; one of the solutions is stable, the other unstable and this characteristic is exchanged while passing the critical value. A simple example is the equation

$$
\dot{x}=\mu x-x^{2}
$$

with critical value $\mu=0$.

Another interesting feature of the lecture notes is Poincaré's analysis of the rings of Saturn. There are three possibilities for the rings: they are solid, liquid or consisting of particles. A solid ring turns out to be unstable for physically realistic values of the parameters. For a liquid ring, Poincaré computes the gravitational potential of a rotating torus; for stability a necessary condition is a very low density of the ring. On the other hand, for stability the pressure should be large enough. These requirements are incompatible. What remains is the possibility of a ring of particles that are separated from each other; this can be a stable configuration.

Interestingly, Christiaan Huygens, who in the seventeenth century was the first to identify the rings of Saturn, drew the same conclusion where he based this on his observations and the fairly restricted physics theory of that time. 


\subsection{Relativity, the Poincaré Group}

In the last decade of the nineteenth century, Poincare got interested in the discussion on electromagnetic field theory for optics and electricity. In that period there were also the intriguing Michelson-Morley experiments trying to demonstrate the motion of the Earth with respect to the ether. Poincaré's first important paper in this field appeared in 1895, the topic kept his interest until his untimely death in 1912.

The Larmor Papers in 1895 In 1895, Poincaré wrote a series of four articles on the theory of Larmor, see [15] and [11, vol. 9]. It contains a lucid discussion of theory and experiments regarding optics and electricity, referring to Larmor, Fresnel, Lorentz, Helmholtz and Hertz. It is of interest that his ideas in 1895 already showed a preparation for the theory of special relativity.

In the preliminary conclusions [11, vol. 9, pp. 409-413], he states that none of the present theories combines both the theoretical requirements of consistency and an explanation of the experiments; the best one seems to be the theory of Lorentz. Altogether, he considers this an unsatisfactory state of affairs. He concludes in 1895 with a revolutionary statement:

The experiments have produced a lot of facts that can be summarised in the following form: it is impossible to demonstrate the absolute motion of matter, or better formulated, the relative motion of substantial matter with respect to the ether; what can be made evident is the motion of substantial matter with respect to substantial matter.

Referring to an experiment by Michelson, he adds that it is not only impossible to demonstrate any motion of matter with respect to the ether but as a second fact that the problem of incorporating the law of 'action is reaction' (Newton's third law) in a description of interaction between matter and ether is unsolved. He concludes that both facts have to be related to each other.

The Paper for Lorentz in 1900 On December 11, 1900, a celebration took place at the university of Leiden on the occasion of the 25 th anniversary of the doctorat of H.A. Lorentz. Poincaré used this occasion to discuss again the problem of the law of 'action is reaction' in electrodynamics (published as [16]). He modifies his earlier remarks that were calling Lorentz' theory the best available but were still mildly critical.

When considering the forces on a collection of electrons bounded in a certain volume and to satisfy the 'action is reaction' law, Poincaré has to assume the presence of non-electric forces. One of the consequences is [11, p. 471]:

As the electromagnetic energy behaves in our point of view like a fluid endowed with inertia, we have to conclude that a device, after having produced electromagnetic energy, radiates in a certain direction, the device has to recoil as a cannon has to recoil when it has launched a projectile.

At this point, in 1900, Poincaré nearly made the step of equating electromagnetic energy with mass, but this step is still too big. A numerical example of the phenomenon shows that the recoil effect is small and difficult to observe. For further understanding one has to consider the motions as relative. If in one dimension, a particle has position $x$ with respect to the observer and $v$ is its velocity in a moving frame of reference, the position in a reference frame indicated by $x^{\prime}$ satisfies the relation:

$$
x^{\prime}=x-v t .
$$


But according to Lorentz we have to introduce local time $t^{\prime}$ by the transformation:

$$
t^{\prime}=t-\frac{v x}{c^{2}}
$$

The explanation for the need of local time is given and repeated extensively in Poincaré's Göttingen lecture [20]. The constant $c$ is the velocity of light, in the reference frame the local time is a second order effect with respect to $1 / c$. For the relative motion in the reference frame, the total energy is not equal to the energy observed at position $x$, it appears that an additional force acts in the reference frame. This looks like a contradiction, but we have to conclude that the energy radiated by the device at the position of the observer is not equal to the energy, in fact bigger than the energy radiated by the device that is placed in a moving frame. The apparent radiation and the apparent recoil energy will make up the difference. In this way, the principle of 'action is reaction' in the theory of Lorentz can be interpreted and saved.

The principle of 'action is reaction' is fundamental in physics, so it is not surprising that the discussion of the principle went on with contributions by Abraham, Planck, Lorentz and others. For references see [11, vol. 9, p. 698].

The Dynamics of the Electron, 1905-1906 A 'comptes rendus' paper [17] in 1905 and its long version in 1906 [18] are concerned with the dynamics of the electron and relativity. Another description and comments can be found in [4].

The paper [17] was submitted on June 5, 1905 which means that it was submitted earlier than Einsteins famous paper on Special Relativity. The five pages, announcing [18], contain the following ideas:

1. All the physical experiments show that the impossibility to show the absolute motion of matter is a general law of nature.

2. Lorentz has proposed to explain this by the contraction of moving bodies in [6]. It explains the present experiments and asks for testing against new experiments.

3. The Lorentz transformation contains several parameters, among which a multiplicative factor function $l(\varepsilon)$. The motion is in the $x$-direction, $\varepsilon$ indicates the ratio of velocity of the body to the velocity of light, $\varepsilon=v / c$, and we have with $k=1 / \sqrt{1-\varepsilon^{2}}$ :

$$
x^{\prime}=k l(x+\varepsilon t), \quad y^{\prime}=l y, \quad z^{\prime}=l z, \quad t^{\prime}=k l(t+\varepsilon x) ;
$$

they are forming a group of transformations. Lorentz gives some arguments to put $l=1$, but Poincaré notes in [17] that the transformation should have invariance for rotation which implies $l=1$.

4. When in motion, an electron can be deformed and compressed as if an exterior force acts.

5. Applying Lorentz' transformation to all forces of nature, one should conclude that the propagation of a gravitational force has to be with the velocity of light. The gravitational attraction of a moving body should take into account the position and velocity of the body to determine the emitted gravitational wave. The difference with Newton's gravitational law is expected to be inverse proportional to the square of the velocity.

There are various statements of Poincare on the principle of relativity. One of them is in [18] ([11, p. 495]):

I semble que cette impossibilité de mettre en évidence expérimentalement le mouvement absolu de la Terre soit une loi générale de la Nature; nous sommes naturellement porté à admettre cette loi, que nous appellerons le Postulat de Rélativité et à l'admettre sans restriction. 
(It seems that this impossibility to establish experimentally the absolute motion of the Earth is a general law of nature; we are of course set towards admitting this law that we will call the Postulate of Relativity and to admit it without restriction.)

The first part of [18] (submitted July 23, 1905) is concerned with the analysis of the Lorentz transformation for a given coordinate system. Consider an electron as the moving body in the $x$-direction and a small sphere around the electron. The comoving sphere is described by the equation

$$
(x-\xi t)^{2}+(y-\eta t)^{2}+(z-\zeta t)^{2}=r^{2} .
$$

Lorentz transformation changes this sphere into an ellipsoid and if the electron charge is invariant, the electrical charge density $\rho$ becomes

$$
\rho^{\prime}=\frac{k}{l^{3}}(\rho+\varepsilon \rho \xi) \text {. }
$$

The continuity equation

$$
\frac{\partial \rho^{\prime}}{\partial t^{\prime}}+\sum \frac{\partial \rho^{\prime} \xi^{\prime}}{\partial x^{\prime}}=0
$$

has been satisfied. This result differs slightly from Lorentz' density $\rho^{\prime}$ in [6]. In the same way, Poincare derives the new electric and magnetic field expressions and in addition the forces acting in the moving frame. Here again, there is a difference with the expressions of Lorentz. The equations for the electric and magnetic fields are the same, the equation for the moving ellipsoid is not. How to explain this difference?

To derive the transformations, one has to use the principle of relativity and the minimalisation of a functional ('le principe de moindre action'). This variational approach leads to Lorentz' expressions for apparent positions and time, but eventually to Poincaré's results described above.

The announcement in [17] that the Lorentz transformations are forming a group is worked out in Sect. 4 of [18]. The group of transformations consists of dilations, boosts and rotations. Combinations of these transformations are permitted and result in a linear transformation, conserving the quadratic form

$$
x^{2}+y^{2}+z^{2}-t^{2} .
$$

By putting $y_{4}=\sqrt{-1} t$ as fourth coordinate, Poincaré introduces [11, vol. 9, p. 542] the metric

$$
d s^{2}=d y_{1}^{2}+d y_{2}^{2}+d y_{3}^{2}+d y_{4}^{2} .
$$

This is the metric, also introduced by Minkowski in 1908.

In the Lie group we can apply the commutator of the infinitesimal generators. If we turn the system over an angle $\pi$ around the $y$-axis, the transformation becomes:

$$
x^{\prime}=k l(x-\varepsilon t), \quad y^{\prime}=l y, \quad z^{\prime}=l z, \quad t^{\prime}=k l(t-\varepsilon x) .
$$

However, in a group, the inverse transformation

$$
x^{\prime}=\frac{k}{l}(x-\varepsilon t), \quad y^{\prime}=\frac{y}{l}, \quad z^{\prime}=\frac{z}{l}, \quad t^{\prime}=\frac{k}{l}(t-\varepsilon x),
$$

should produce identical results. It follows that $l=1 / l$ or $l=1(l=-1$ does not correspond with a new physical configuration). 
The formulation of the Lorentz group, now also called the Poincaré group, and the establishing of $l=1$ belong to the permanent results of the paper on the dynamics of the electron.

In the last section of [18] the consequences for the theory of gravitation are analysed. The change with respect to the classical theory will be that gravitation will not only depend on position and mass, but also on the velocity of the mass at time $t_{0}$. In addition, as gravitation takes time to travel, we have to take into account the position and velocity at time $t_{0}+t$ when the propagation of the force started; so $t$ will be negative. In addition Poincaré notes that the substitutions of the group do not change the quadratic form (2). Points in space have coordinates $x, y, z, \sqrt{-1} t$; applying the coordinate transformations and the invariants, the only consistent choice that does not lead to contradictions is that gravitation propagates with the velocity of light. As the deviations from Newtons laws are quadratic in the ratio of velocity of the body and the velocity of light, the effect will be difficult to observe.

\subsection{Relativity: The Priority Question}

A detailed account of the development of the theory of relativity can be found in the second volume of Whittaker's history of the theory of aether and electricity [27], in particular in the chapter "The relativity theory of Poincaré and Lorentz". The main priority controversy regarding the new mechanics, replacing Newtonian classical mechanics by relativity, is on Special Relativity with prominent candidates Einstein, Lorentz and Poincaré. The relativity of motion itself was studied and formulated already by Galilei and Huygens, but they still assumed the existence of an absolute reference frame for motion. Its perspective changed drastically by the experiments around 1900 showing that the velocity of light is independent of the inertial system chosen by the observer. H.A. Lorentz (1853-1928) used this constancy of the velocity of light in each inertial system as the basis of his mechanics. It is the maximum velocity that can be observed and to allow for this he made the brilliant assumption that size and mass of a body are dependent on the velocity in a given inertial system. His formula for the so-called Lorentz-contraction gives this relation explicitly. In addition Lorentz introduced the fundamental concept of local time, which means time as dependent on position and velocity in a given inertial system. So, like size and mass, time has no absolute meaning, there is no absolute reference frame for motion.

Poincaré noted already in 1900 that radiation could be considered as a fictitious fluid with the behaviour of an equivalent mass, implying $E=m c^{2}$, but without detailed calculations. He derived this interpretation from Lorentz's 'theory of electrons' which incorporated Maxwell's radiation pressure. It seems remarkable that Poincaré, who was always correct and even generous in citing people, did not mention Einstein in his 1909 lecture in Göttingen [20]. The explanation is probably as follows.

Most physicists and mathematicians of that time considered H.A. Lorentz as the prominent contributor to the theory of the new mechanics, now called Special Relativity. It is typical that still in 1913 Darboux writes [1] that Poincaré discussed 'the mechanics of Lorentz'. There are indications however that Lorentz considered his observations as provisional hypotheses whereas Einstein presented a complete and new vision of physical reality, certainly when in 1916 he formulated general relativity. In 1927 Lorentz [9] formulated it at a conference as follows:

I considered my time transformation only as a heuristic working hypothesis. So the theory of relativity is really solely Einstein's work. And there can be no doubt that he would have conceived it even if the work of all his predecessors in the theory of this field had not been done at all. His work is in this respect independent of the previous theories. 
Regarding the theory of Special Relativity, this was maybe overly generous.

Poincaré and Lorentz certainly show an ambivalence when mentioning the ether as a matter of fact in their writings, they seem to be reluctant to ignore or to do without it; Poincaré's also uses the ether as a working hypothesis in his philosophical books which were aimed at a wide public. In his lecture [8] Lorentz put it like this:

Why can we not speak of the ether instead of vacuum? Space and time are not symmetric, a material point can at different times be at the same spot, but not in different places at the same time.

Still, in a discussion of priorities, the fundamental contributions of Lorentz to the formulation of special relativity theory, for instance his transformation formulae and the concept of local time, should be recognised together with Poincaré's contributions: his formulation of the Lorentz or Poincaré group and the principle of relativity. In a way Lorentz' 1927 formulation given above, should be supplemented with his appreciation of Poincaré's 'dynamics of the electron' papers, given in [7, p. 298]:

Poincaré, on the other hand, has obtained a perfect invariance of the equations of electrodynamics and he has formulated the 'relativity postulate', in terms that he was the first to use.

In a lecture at the Royal Academy of Sciences in Amsterdam, Lorentz put it in 1915 as follows [8]:

I could point out to you [if I had more time, F.V.] how Poincare in his study of the dynamics of the electron, about the same time as Einstein, has formulated many ideas that are characteristic for his theory, and also has formulated what he calls "le postulat de relativité".

Einstein, when describing in 1949 the development of relativity [2], mentions many scientists, in particular Lorentz, but does not mention Poincaré.

Acknowledgements Remarks and questions from Giuseppe Gaeta and Antonio Degasperis were helpful in writing this paper.

Open Access This article is distributed under the terms of the Creative Commons Attribution License which permits any use, distribution, and reproduction in any medium, provided the original author(s) and the source are credited.

\section{References}

1. Darboux, G.: Éloge historique d'Henri Poincaré. In: [11], vol. 2, pp. VII-LXXI. Presented as a lecture on December 5, 1913

2. Einstein, A.: The theory of relativity. In: Out of My Later Years. Philosophical Library, New York (1950)

3. Kirillov, O.N., Verhulst, F.: Paradoxes of dissipation-induced destabilization or who opened Whitney's umbrella. Z. Angew. Math. Mech. 90, 462-488 (2010)

4. Le Bellac, M.: The Poincaré group. In: Charpentier, E., Ghys, E., Lesne, A. (eds.) The Scientific Legacy of Poincaré. History of Mathematics, vol. 36, pp. 329-350. AMS, Providence (2010)

5. Lebovitz, N.R.: The mathematical development of the classical ellipsoids. Int. J. Eng. Sci. 36, 1407-1420 (1998)

6. Lorentz, H.A.: Electromagnetic phenomena in a system moving with any velocity smaller than that of light. In: Proc. Royal Academy of Sciences, Amsterdam, May 27 (1904). Reprinted in Collected Papers, vol. 5, pp. 172-197, Martinus Nijhoff, The Hague (1939)

7. Lorentz, H.A.: Deux mémoires de Henri Poincaré dans la physique mathématique. Acta Math. 38, 293308 (1914). Reprinted in Collected Papers, vol. 8, pp. 258-273, Matinus Nijhoff, The Hague (1934) 
8. Lorentz, H.A.: De lichtaether en het relativiteitsbeginsel (The light-ether and the principle of relativity). In: Lecture for the Royal Academy of Sciences, Amsterdam, April 24 (1915). Reprinted in Collected Papers, vol. 9, pp. 233-243, Martinus Nijhoff, The Hague (1939)

9. Lorentz, H.A.: Conference on the Michelson-Morley experiment. Astrophys. J. 68, 345-351 (1928)

10. Mawhin, J.: Henri Poincaré and the partial differential equations of mathematical physics. In: Charpentier, E., Ghys, E., Lesne, A. (eds.) The Scientific Legacy of Poincaré. History of Mathematics, vol. 36, pp. 373-391. AMS, Providence (2010)

11. Poincaré, H.: Oeuvres de Henri Poincaré Publiées sous les Auspices de l'Académie des Sciences, vols. 112. Gauthier-Villars, Paris (1916-1954)

12. Poincaré, H.: Sur les équations aux dérivées partielles de la physique mathématique. Am. J. Math., 12, 211-294 (1890). Also in [11], vol. 9, pp. 28-113

13. Poincaré, H.: Sur léquation des vibrations d'une membrane. C. R. Acad. Sci. Paris 118(12), 447-451 (1894). Also in [11], vol. 9, pp. 119-122

14. Poincaré, H.: Sur les équations de la physique mathématique. Rend. Circ. Mat. Palermo 8, 57-155 (1894). Also in [11], vol. 9, pp. 123-196

15. Poincaré, H.: A propos de la théorie de M. Larmor. L’Éclairage électrique, t. 3, pp. 5-13, pp. 289-295, t. 5, pp. 5-14, pp. 385-392, 1895. Also in [11], vol. 9, pp. 369-426

16. Poincaré, H.: La théorie de Lorentz et la principe de réaction. In: Archives Néerlandaises des Sciences Exactes et Naturelles, 2e Série, vol. 5, pp. 252-278 (1895). Also in [11], vol. 9, pp. 464-488

17. Poincaré, H.: Sur la dynamique de l'électron. C. R. Acad. Sci. 140, 1504-1508 (1905). Also in [11], vol. 9, pp. 489-493

18. Poincaré, H.: Sur la dynamique de l'électron. Rend. Circ. Mat. Palermo 21, 129-176 (1906). Also in [11], vol. 9, pp. 494-550

19. Poincaré, H.: Les Méthodes Nouvelles de la Mécanique Célèste, 3 vols. Gauthier-Villars, Paris $(1892 / 1893 / 1899)$

20. Poincaré, H.: Sechs Vorträge aus der reinen Mathematik und mathematischen Physik. In: Mathematische Vorlesungen an der Universität Göttingen: IV, auf Einladung der Wolfskehl-Kommission der Königlichen Gesellschaft der Wissenschaften gehalten zu Göttingen vom 22-28 April 1909. Teubner, Leipzig (1910)

21. Sanders, J.A., Verhulst, F., Murdock, J.: Averaging Methods in Nonlinear Dynamical Systems, 2nd edn. Applied Mathematical Sciences, vol. 59. Springer, Berlin (2007)

22. Tchebycheff, P.L.: Oeuvres, vol. 2 (1907). A. Markoff and N. Sonin (eds.), St. Petersburg

23. Thomson, W., Tait, P.G.: Treatise on Natural Philosophy. Cambridge University Press, Cambridge (1883)

24. Van der Aa, E., De Winkel, M.: Hamiltonian systems in $1: 2: \omega(\omega=5$ or 6$)$ resonance. Int. J. NonLinear Mech. 29, 261-270 (1994)

25. Van der Aa, E., Verhulst, F.: Asymptotic integrability and periodic solutions of a Hamiltonian system in (1:2:2)-resonance. SIAM J. Math. Anal. 15, 890-911 (1984)

26. Verhulst, F.: Henri Poincaré, Impatient Genius. Springer, Berlin (2012)

27. Whittaker, E.: A History of the Theories of Aether and Electricity, the Modern Theories 1900-1926. Thomas Nelson and Sons, London (1953) 